\title{
Chamula: pueblo de migrantes en Los Altos de Chiapas
}

\author{
Chamula: a migrant town in Los Altos, Chiapas
}

\section{Gonzalo Coporo Quintana* \\ Daniel Villafuerte Solís**}

\begin{abstract}
Resumen. Este artículo analiza la migración internacional en Chamula, un municipio indígena de Los Altos de Chiapas, cuyas condiciones de vida material de su población y su historia particular lo han llevado a la búsqueda de alternativas de sobrevivencia, primero como jornaleros en las fincas cafetaleras del Soconusco y colonizadores en distintos puntos de la geografía chiapaneca, y luego a las zonas urbanas de los estados del sur de México, el Distrito Federal y el Estado de México, y en los últimos años a las entidades federativas del norte del país y a Estados Unidos. El objetivo de este trabajo consiste en señalar que la migración de la población de Chamula es indicativa de un cambio en las tendencias de la migración internacional en México, donde la profundización del modelo económico neoliberal condiciona a los sectores más pobres a que se desplacen más allá de las fronteras nacionales.
\end{abstract}

Palabras clave. Migración indígena, migración internacional, pobreza, exclusión social y desarrollo.

\begin{abstract}
This article analyzes international migration in Chamula, an indigenous municipality of Los Altos, Chiapas, where the material conditions of life for its people and its particular history have led to a search for alternative strategies for survival, first as laborers in the coffee plantations of Soconusco and settlers in various parts of Chiapan territory, and later in the urban areas of the southern states of Mexico, the Federal District and the State of Mexico, and in recent years in the Northern Mexican states and to the United States. The aim of this work is to show that the migration of the Chamula population is indicative of a change in the international migration tendencies in Mexico, where the entrenchment of the neoliberal economic model is leading the poorest sectors of Mexican society to be displaced beyond national borders.
\end{abstract}

Keywords. Indigenous migration, international migration, poverty, social exclusion and development.

* Centro de Estudios para el Desarrollo Municipal y Políticas Públicas, Universidad Autónoma de Chiapas.

** Centro de Estudios Superiores de México y Centroamérica, Universidad de Ciencias y Artes de Chiapas. 



\section{Introducción}

Chamula ocupa un lugar emblemático en la historia y en la geografía de Chiapas. Su participación en las revueltas indígenas de mediados del siglo XIX y principios del XX; el fuerte vínculo que estableció y mantiene hasta el presente con el Partido Revolucionario Institucional (PRI) — que Rus (1998) atinadamente caracterizó como la «Comunidad Revolucionaria Institucional»—, constituyen evidencias de la presencia de esta comunidad en la historia de Chiapas. Todos los candidatos que aspiran a ocupar la gubernatura del estado y la presidencia de la república indefectiblemente incluyen en su itinerario la visita de San Juan Chamula, cabecera del mismo municipio.

El pueblo Chamula ha sido objeto de numerosos estudios antropológicos, agroecológicos, sociales, económicos y culturales. Una obra que quizá retrata con nitidez el interés por los chamulas es Juan Pérez Jolote, escrita por Ricardo Pozas en 1948, es una obra que oscila entre la antropología y la literatura, por medio del personaje proyecta una parte del mundo de vida de los chamulas. Su sistema de cargos y las festividades, especialmente el carnaval ha atraído la atención de numerosos estudiantes y profesionales de las ciencias sociales. Su estrecha relación con las fincas cafetaleras del Soconusco hasta la mitad de los 1970, a través de la venta de fuerza de trabajo mediante un sistema de enganche, condujo en 1936 a la creación del sindicato de trabajadores indígenas (García de León, 1985) asociado al Partido Nacional Revolucionario (PNR) registró una membresía de 30 mil jornaleros indígenas. ${ }^{1}$

Hasta la primera mitad de la década de 1980 este fue un tema de preocupación en la agenda de investigación social. El conflicto político-religioso y las expulsiones de grupos protestantes merecieron algún tiempo el interés de las ciencias sociales, hecho que propició una extensa bibliografía histórica y antropológica que sería demasiado prolijo mencionar en este breve escrito (López, 1992; Pérez, 1994; Morquecho, 1992; Betancourt, 1977). Hoy, el fenómeno de la migración internacional ocupa un espacio cada vez más importante en los estudios sociales,

\footnotetext{
${ }^{1}$ Diez años después, en la posguerra, y frente a la crisis del movimiento campesino, el sindicato de trabajadores indígenas asume de forma plena la tarea que de alguna manera se ocultaba detrás de su carácter reivindicativo de los derechos de los jornaleros, en ese sentido Paniagua comenta: «El sindicato cumple actualmente la función de los enganchadores: asegura el reclutamiento de la fuerza de trabajo necesario para las plantaciones en época de cosecha» (Paniagua, 1983:52).
} 
pero todavía hay mucho que decir, en concreto respecto a su impacto en las familias, la cultura y los sistemas de organización comunitarios.

En los últimos veinte años, grupos de chamulas han pasado a formar parte del paisaje humano de varios de los estados de la Unión Americana, especialmente de California, Georgia y Florida; sin embargo, existen muy pocos trabajos sobre las características que asume la migración internacional de la población Chamula. Uno de los estudios pioneros es el de Rus y Rus (2008) La migración de trabajadores indígenas de Los Altos de Chiapas a Estados Unidos, 2001-2005: el caso de San Juan Chamula, que ilustra grosso modo la dinámica migratoria de dicho municipio. En ese contexto, el presente artículo se suma a lo ya realizado, con el propósito de brindar datos en torno a las condiciones materiales y la migración, a partir del análisis de dos comunidades representativas de lo que ocurre en el ámbito de la geografía municipal: Nichnamtic y Mukinaló, ${ }^{2}$ ubicadas en el oriente del municipio de Chamula. Comprende tres partes: la primera muestra una caracterización de la dinámica demográfica y la base productiva del municipio y las localidades de estudio, la segunda examina el fenómeno de la pobreza y la exclusión social en el ámbito municipal y su manifestación concreta en Nichnamtic y Mukinaló, la tercera aborda la migración en tales localidades articulada con el mundo material y económico; el texto concluye con algunas consideraciones finales.

\section{Dinámica demográfica y base productiva}

Chamula forma parte de Los Altos de Chiapas, una región integrada por 18 municipios $^{3}$ con una extensión territorial de 4 mil 725 kilómetros cuadrados, según el Censo de Población y Vivienda 2010, cuenta con una población regional de 627 mil 569 habitantes (INEGI, 2010), el 30 por ciento se concentra en el municipio de San Cristóbal de Las Casas, localidad que históricamente ha sido un centro de atracción de las comunidades indígenas de la región, pues condensa

${ }^{2}$ Mukinaló es el nombre Tsotsil de la localidad Las Ollas. Mukinaló es la forma como se escucha Mukinalvo, significa agua enterrada o agua subterránea. Nichnamtic, significa flor de laguna.

${ }^{3}$ La regionalización en que se basa este artículo es la que existía hasta el año 2012, es decir la que incluía 18 municipios. La aclaración es pertinente en tanto que a partir del año 2013 la regionalización del estado de Chiapas quedó constituida, según decreto oficial, por 15 regiones, siendo ahora Los Altos, La Región V Altos. Tsotsil-Tseltal, integrada por 17 municipios, queda fuera el municipio de Las Rosas. 
los servicios económicos de salud, educación, transporte, heredada de su estatus de capital de la Provincia de Las Chiapas hasta el año de 1892. Parafraseando a Marroquín (1978), San Cristóbal de Las Casas ha sido la ciudad mercado de Los Altos, cuya trasformación dio paso en años recientes a la proliferación de establecimientos comerciales de capital nacional y extranjero.

El municipio de Chamula posee una extensión territorial ${ }^{4}$ de 341 kilómetros cuadrados y una población de 76 mil 941 habitantes, lo que arroja una densidad demográfica de 225 habitantes por kilómetro cuadrado. Se trata de una población rural e indígena, con alto porcentaje de monolingüismo. De acuerdo con los resultados del Censo de Población y Vivienda 2010, el 99 por ciento de la población de tres años y más habla lengua indígena y 60 por ciento es monolingüe. Esta característica le imprime un carácter particular a la migración hacia Estados Unidos, además representa un choque cultural en su expresión más amplia, no sólo por su origen rural sino por su lengua de origen maya.

Para entender el proceso migratorio actual es necesario volver la vista atrás y observar la dinámica socioeconómica y demográfica desde 1970 hasta 2010, ${ }^{5}$ dado que a partir de esa época se observa un punto de inflexión en la historia particular del municipio de Chamula. Cabe mencionar que el crecimiento de la población por sí mismo conduce a determinadas conclusiones sin tener en cuenta el sustrato material sobre el que la población se reproduce, en específico la tierra, por tratarse de una comunidad rural, con fuerte trayectoria de organización comunitaria. Comenzaremos por exponer un cuadro de la población de las cuatro décadas que comprende el periodo mencionado, con todas las limitaciones de la información censal, pero indicativo de los cambios.

En la década de 1970, mientras el crecimiento de la población chiapaneca fue de casi 2.9 por ciento, Chamula manifestó un incremento inferior al 1 por ciento, lo que revela la fuerte migración interna e interestatal del periodo caracterizado por la construcción de grandes centrales hidroeléctricas, el boom petrolero (Angulo, 2007) y el desarrollo del megaproyecto turístico de Cancún; a ello habría que añadir los grandes conflictos político-religiosos que terminaron con la

${ }^{4}$ El año base de la superficie continental del INEGI es del año 2005, según se aprecia en la información de su página web. México en Cifras. Los datos del INEGI no están exentos de errores, dado que en 1970 tenía una superficie continental para Chiapas de 73 mil 887 kilómetros cuadrados, pero otras fuentes registraban poco más de 74 mil kilómetros cuadrados y otras más indicaban una extensión mayor a los 75 mil kilómetros cuadrados.

${ }^{5}$ Lo cual ocurre dentro del contexto de los cambios estructurales que vive el país y el estado de Chiapas. 
expulsión de varios miles de habitantes de diversas comunidades del municipio (Morquecho, 1992; Betancourt, 1977).

Para los 1980 incrementa de manera desproporcionada el crecimiento de la población de Chiapas, sin que exista algún evento extraordinario. Cómo explicar que mientras en la década precedente la población creció en casi 33 por ciento y en la de 1990 alcanzó una cifra cercana al 59 por ciento. Concerniente a Chamula es aún más inverosímil, ya que mientras en los 1970 aumentó en menos de 7 por ciento, en la década posterior lo hizo en 65 por ciento. Expresado en tasas de crecimiento anual, Chamula pasó de menos del uno por ciento en 1980 a más del 5 por ciento en 1990. Por ende, consideramos que la cifra censal de 1990 no es útil para observar el comportamiento de la población. ${ }^{6}$ Indudablemente la información intercensal de 1980-1990 del INEGI posee serios sesgos y no permite hacer una lectura cercana a la realidad en lo que respecta a Chiapas y Chamula.

\section{CUADRO 1}

Población total, densidad demográfica y tasa media anual de crecimiento, comparativo entre Chiapas y Chamula

\begin{tabular}{c|ccc|ccc} 
Años & \multicolumn{3}{|c}{ Chiapas } & \multicolumn{3}{c}{ Chamula } \\
& Población & Densidad & TMCA & Población & Densidad & TMCA \\
\hline 1970 & 1569053 & 21.41 & ------ & 29357 & 86.08 & ------- \\
1980 & 2084717 & 28.45 & 2.88 & 31364 & 91.96 & 0.66 \\
1990 & 3310496 & 43.81 & 4.41 & 51757 & 151.75 & 5.14 \\
2000 & 3920892 & 53.50 & 2.02 & 59005 & 173.00 & 1.32 \\
2010 & 4796580 & 65.45 & 2.04 & 76941 & 223.66 & 2.69 \\
\hline
\end{tabular}

Fuente: Censos generales de población y vivienda.

En los 1990 inicia un periodo de veinte años con una tendencia visible a la baja en el ritmo de crecimiento de la población chiapaneca, en concreto la de Chamula. Para Chiapas, en el periodo intercensal 1990-2000 la tasa de crecimiento es ligeramente superior al 2 por ciento y para Chamula 1.3 por ciento. Es una etapa en esencia importante porque concurren diversos fenómenos que tendrán impactos significativos en la movilidad de la población: 1) crisis en el sector

${ }^{6}$ En comunicación personal con Villafuerte en marzo de 2013, menciona que el nuevo gobierno de Chiapas, que asumió el poder en diciembre de 1988, trabajó a favor de un incremento en la población para favorecer la transferencia de recursos de la federación a la entidad. 
agropecuario que afectó severamente a los precios de los principales productos, entre ellos café, plátano y maíz. 2) Levantamiento armado del Ejército Zapatista de Liberación Nacional (EZLN). 3) Reestructuración de las instituciones, en particular las vinculadas al sector rural, que provocó disminución de los apoyos, subsidios y créditos. 4) La crisis económica y financiera que se expresó en la macro devaluación del peso y la fuga de capitales desde diciembre de 1994.

Esta década fue particularmente trágica para Chiapas porque además de la implementación del modelo económico neoliberal, con todas sus consecuencias sociales, tuvieron lugar eventos hidrometereológicos —el huracán Mitch en 1998 que afectaron grandes zonas del estado e hicieron que la producción agrícola disminuyera, además que se reorientaron recursos financieros para mitigar los desastres en la Sierra, el Soconusco y el Centro, desatendiendo a otras regiones como Los Altos donde se ubica el municipio estudiado. En la conjunción de los eventos referidos, se hace visible la migración internacional, de modo que hacia el año 2000 ya se tenían registros consolidados sobre remesas y había crecido el número de «agencias de viajes» que hacían el recorrido por el Pacífico y por el Golfo, hasta la frontera con Estados Unidos (López; Molina y Villafuerte, 2010).

En el periodo intercensal 2000-2010 se advierte que el ritmo de crecimiento de la población chiapaneca se mantiene sin cambios con relación a la década anterior; no obstante, Chamula presenta un giro notorio, la tasa de crecimiento aumenta prácticamente al doble (de 1.32 a 2.69 por ciento). خQué explicación merece este cambio tan significativo en medio de un creciente flujo migratorio? En ambos periodos censales la tasa de fecundidad se mantuvo en 2.88 hijos, en tanto que a nivel de Chiapas la fecundidad bajó de 2.73 a 2.52 hijos en promedio. Por otra parte, el crecimiento de la población de las últimas cuatro décadas ha provocado el aumento de la densidad de población de forma desproporcionada, si se toma en cuenta que la tierra es un recurso crítico. Mientras la densidad demográfica en Chamula en 1970 era cuatro veces mayor que la de Chiapas, en 2010 la proporción había bajado a 3.5 veces, aun así el crecimiento absoluto de la población — que se multiplicó por 2.6 - se convirtió en un serio problema frente a la escasez de recursos productivos.

Como hemos indicado, la población de Chamula es fundamentalmente rural, cuyos asentamientos están dispersos por la geografía municipal; las cifras del Censo de Población y Vivienda 2010 revelan la existencia de 144 localidades y poco más de 63 por ciento de la población vivía en 109 localidades (75.7 por ciento del total) de entre 100 y 999 habitantes, lo que confirma el nivel de ruralidad del 
municipio. Sólo una localidad, San Juan Chamula, la cabecera municipal, contaba con 3 mil 329 habitantes.

En concordancia con su condición de ruralidad, se constató que en 2010, el 63 por ciento de la población ocupada $\left(24\right.$ mil 481) ${ }^{7}$ se ubicó en las actividades agropecuarias, el resto se distribuyó casi de manera equitativa entre el sector secundario y el terciario. Con base en el VIII Censo Agropecuario de 2007, se registraron 15 mil 244 unidades de producción rural, con una superficie de 26 mil 938 hectáreas, lo que arroja un promedio de 1.7 hectáreas por persona ocupada en el sector. Este dato es indicativo de las fuertes limitaciones de la población para autoabastecerse de alimentos y obtener ingresos suficientes que le permitan mantener la reproducción familiar. El problema es todavía más crítico si se considera que de la superficie censada únicamente 18 mil 225 hectáreas se clasificaban como de labor (poco más de 6 mil con pastos y agostadero y más de mil 863 con selva), con lo que se tendría sólo 0.74 hectáreas de labor por persona ocupada. Al creciente minifundismo debe agregarse el deterioro de las tierras por efectos de la erosión y el manejo inadecuado, los cuales se reflejan en la cantidad de producto obtenido (INEGI, 2007).

Al relacionar lo anterior con algunos resultados de la investigación (Coporo, 2013) realizada en las localidades de Nichnamtic y Mukinaló, ${ }^{8}$ ubicadas en la zona sur-oriente del municipio de Chamula, ${ }^{9}$ representativas de la migración internacional (según la información recabada en los cuestionarios y entrevistas del trabajo de campo realizado de 2009 a 2012) ${ }_{1}^{10}$ encontramos, tal como se aprecia en el cuadro 2, que la superficie cultivada con el principal producto para la alimentación, el maíz, es muy pequeña; en tanto que cincuenta encuestados declararon contar con una superficie de entre $40 \mathrm{~m}^{2}$ a 0.5 hectáreas (esta última superficie es fraccionada, es decir, no se encuentra en el mismo lugar). Es evidente que no es posible conseguir el sustento de la familia, por lo que son obligados a salir de la

${ }^{7}$ La población económicamente activa fue de 25 mil 29 personas, lo que revela una tasa muy baja de desempleo; sin embargo, el problema principal es el subempleo, asociado a la precariedad laboral que en buena medida se refleja en la proporción de fuerza de trabajo asalariada y el reducido nivel de ingreso cuyo referente es el alto nivel de pobreza.

${ }^{8}$ Cada localidad cuenta con un aproximado de 350 familias.

${ }^{9}$ La zona oriente adquirió relevancia porque a decir de Rus (2009) fue importante en la producción y distribución de Posch (aguardiente), pero también porque aquí surgió un sector de la población, particularmente comerciantes y maestros, que empezó a mostrar su inconformidad con las autoridades tradicionales en la década de los 1970, lo que propició el conflicto político religioso de esa década.

${ }^{10}$ Se aplicaron 230 cuestionarios en las dos localidades de estudio: 103 en Nichnamtic y 127 en Mukinaló (Coporo, 2013). 
comunidad y emplearse como asalariados. En el otro extremo, sólo dos encuestados declararon contar con $50 \mathrm{mil} \mathrm{m}^{2}$ (5 hectáreas).

\section{CUADRO 2}

Superficie cultivada con maíz y número de campesinos

\begin{tabular}{ccc}
$\begin{array}{c}\text { Grupos de superficie } \\
\text { (metros cuadrados) }\end{array}$ & $\begin{array}{c}\text { Número de } \\
\text { campesinos }\end{array}$ & $\begin{array}{c}\text { Superficie total } \\
\text { (metros cuadrados) }\end{array}$ \\
\hline De 40 a 5000 & 50 & 124153 \\
De 10000 a 15000 & 28 & 280000 \\
De 20000 a 30000 & 8 & 200000 \\
De 50000 & 2 & 100000 \\
\hline Total & 88 & 704153
\end{tabular}

Fuente: Coporo, 2013.

En otras palabras, si consideramos el número total de encuestados que respondieron, tendremos que 88 campesinos reúnen una superficie de 70.4 hectáreas, osea un promedio de 0.8 hectáreas por persona. Aunque si tomamos únicamente al grupo más numeroso tendríamos 0.24 hectáreas por campesino, lo que refleja la precariedad estructural de los productores indígenas de las comunidades estudiadas.

Atendiendo las superficies cultivadas, declaradas por los encuestados, la producción de maíz se resume de la siguiente manera: 50 por ciento produce entre diez y veinte costales de maíz al año, 23.8 por ciento entre uno y ocho costales, y sólo 8 por ciento produjo más de veinte costales, el resto no respondió. El costal de maíz contiene aproximadamente 85 kilogramos, lo que significa que ese 50 por ciento de los que respondieron obtuvieron un volumen promedio de 1.3 toneladas al año, si estimamos que el consumo promedio de maíz para una familia de cuatro miembros es de aproximadamente 3 kilogramos diarios, equivalente en tortillas, apenas si estaría cubriendo una parte de sus necesidades. El segmento que representó 23.8 por ciento debe acudir al mercado para satisfacer el consumo de este grano básico.

Aparte del cultivo del maíz, los campesinos siembran frijol, calabaza, haba, col, chícharo y papa. Con excepción de los tres últimos, el resto se asocia con el cultivo del maíz. Se trata del sistema de milpa — típica de una parcela campesina-, donde el maíz siempre aparece asociado con los cultivos mencionados, práctica que mantiene la racionalidad campesina que busca aprovechar los escasos 
recursos con que cuenta. La col, el chícharo y la papa son cultivos de autosubsistencia destinados en esencia al mercado regional. En el caso de las comunidades estudiadas, sólo diez campesinos declararon cultivar papa: uno sembró 2 hectáreas, tres 1 , dos 0.5 , uno 0.25 y el resto osciló entre 100 y 500 metros cuadrados.

Asimismo, es común en Chamula, y en otros municipios indígenas de Los Altos de Chiapas, la práctica de la ganadería menor, en la que sobresale la cría de borregos y aves de corral, siendo marginal la cría de cerdos, conejos y ganado vacuno. De manera que las dos primeras son las actividades de mayor importancia, su cuidado dentro de la división sexual del trabajo, corresponde a mujeres y niños:

La mujer indígena establece un vínculo afectivo con cada uno de sus borregos, éstos son parte de su actividad cotidiana, su responsabilidad ante la unidad familiar. Los borregos no sólo proporcionan la materia prima para elaborar ropa tradicional, la que identifica al grupo étnico, sino también son, según las creencias locales, el reflejo de la actitud de Dios hacia la Familia (Perezgrovas, 1999:101).

Hoy, en el marco de las nuevas articulaciones con el mercado laboral de las comunidades indígenas es común observar a las mujeres que pastorean los borregos portando un teléfono celular, que sirve de dispositivo de comunicación con sus maridos o hijos que trabajan en Estados Unidos, también es común la presencia de mujeres en las instituciones bancarias de San Cristóbal de Las Casas cobrando las remesas que envían sus familiares.

La cría de borregos es pues una actividad que complementa el ingreso de las familias campesinas-indígenas y contribuye a su reproducción. De los borregos se aprovecha básicamente la lana que las mujeres venden en el mercado de San Juan Chamula y San Cristóbal de Las Casas, que se destina a la confección de las prendas de vestir de hombres y mujeres. La carne no se consume porque «así está dispuesto por la costumbre, así lo dicen los antepasados, de manera que ni se puede ni se debe hacerlo» (Perezgrovas, 1999:194).

\section{La pobreza y el doble carácter de la exclusión social}

Chamula se encuentra en la lista de municipios con mayor pobreza y marginación del país. Forma parte de los 125 municipios con menor Índice de Desarrollo Humano y de los 55 municipios incluidos en la lista de la Cruzada 
Nacional contra el Hambre, con 94.8 por ciento de su población en pobreza, de los cuales 69.7 por ciento en pobreza extrema y 31 por ciento con carencia de alimentación (CONEVAL, 2010).

De acuerdo con el Censo General de Población 2010, de las 43 mil 445 personas de 15 años y más, sólo 22 mil 238 (51 por ciento) contaban con primaria completa, y 17 mil 646 (40.9 por ciento) no contaba con escolaridad. El rezago social que padece la población de Chamula es alarmante, las estimaciones realizas por CONEVAL (2010), con fundamento en la ENIGH y el Censo de Población y Vivienda 2010, revelan que 55 por ciento de la población muestra rezago educativo, 98.4 por ciento no posee seguridad social, 96.5 por ciento carece de servicios básicos en la vivienda y 94.8 por ciento tiene ingresos inferior a la línea de bienestar.

La condición étnica de los habitantes del municipio de Chamula tiene un peso significativo en la explicación de la exclusión social. El acceso a los servicios básicos (agua, electricidad, educación, salud, entre otros) están mediados por la condición étnica, el racismo imperante en la sociedad, en específico en la clase gobernante, hace que la dotación de los servicios manifieste un desfase histórico con respecto a otras regiones. En la época del estado de bienestar y del desarrollismo, las políticas gubernamentales aplicadas a través de instituciones, como el Instituto Nacional Indigenista, pretendían «integrar» a la nación a los grupos étnicos que están al margen de los beneficios del desarrollo.

En los 1970, cuando el estado de bienestar entraba en una fase inicial de crisis, las comunidades indígenas de Los Altos de Chiapas fueron objeto de programas especiales para evitar el deterioro económico y social que estaban experimentando, sobresale el Programa de Desarrollo Socioeconómico de Los Altos de Chiapas (PRODESCH) correspondiente al sexenio del gobernador Manuel Velasco Suárez $\mathrm{y}$ al que se refiere en los siguientes términos:

El presidente Echeverría, compenetrado de los asuntos que afectan a nuestros indígenas, ordenó el $1^{\circ}$ de enero del presente año, se diera cumplimiento al Acuerdo Básico firmado con los Organismos Internacionales de las Naciones Unidas y así iniciaron las actividades de nuestro Programa de Desarrollo Socioeconómico de los Altos de Chiapas dirigido para beneficiar a 280,000 habitantes de 21 municipios en un área de 5,500 kilómetros cuadrados, con un Plan de Operaciones preparado por la UNICEF enfocado a coordinar las acciones del Gobierno del Estado con 13 Dependencias Federales y con la Cooperación Internacional (Velasco, 1972:42). 
En la época del neoliberalismo, la población campesina e indígena dejó de ser una preocupación del Estado, que sólo procuró ofrecer los mínimos recursos para evitar que la población se muriera de hambre o se levantara en armas. El 1 de enero de 1994, con el levantamiento del EZLN, quedó al descubierto la precariedad material de los pueblos indígenas de Chiapas, algunas comunidades del municipio de Chamula se sumaron al movimiento neozapatista, y se inauguró una época muy compleja en el escenario económico, social, cultural y religioso, en la que las identidades se fraccionaron. En el contexto del modelo neoliberal, el gasto público se orienta a sectores considerados social y económicamente rentables, y para los pueblos indígenas se destinan recursos marginales, ello se refleja por ejemplo en el gasto público del gobierno de Chiapas durante los últimos 12 años.

A más de cuatro décadas del PRODESCH, que prometía sacar de la marginación a la población indígena, esto no ha cambiado, sobre todo en la región que nos ocupa, dado que ésta observa una extensión de la geografía de la pobreza. En la región de Los Altos habitan 627 mil 569 personas (INEGI, 2010), distribuidos en 18 municipios, es decir, 124 por ciento más con respecto a la cifra indicada por el gobierno del estado en 1972. En ese sentido, las estadísticas de CONEVAL (2010) respaldan la siguiente aseveración: en el año 2010, el citado organismo estimó para Chiapas una población de 3 millones 784 mil 967 personas en situación de pobreza, equivalente a 78.5 por ciento; sin embargo, para la población de Los Altos, el promedio alcanzó un porcentaje de 85.2, con excepción de San Cristóbal de Las Casas, cuya cifra fue de 66.1 por ciento, encontramos que la mitad de municipios se hallaba por arriba del 95 por ciento, Chamula registró 94.8 por ciento.

Por otra parte, el cálculo del Índice de Desarrollo Humano del Programa de Desarrollo Humano de las Naciones Unidas (2013) para el año 2010, confirman la situación de pobreza de más del 50 por ciento de los municipios con menor Índice de Desarrollo Humano (IDH). En este documento se registra que tres municipios de Los Altos de Chiapas tienen el IDH más bajo de Chiapas y forman parte de la lista nacional de municipios con menor desarrollo humano: Chamula, Mitontic y Zinacantán, cuyos índices ( $0.446 ; 0.448$ y 0.461) son equiparables a la de ciertos países africanos como Guinea Bissau, Liberia o Zambia.

Finalmente, la información del Censo de Población y Vivienda de 2010, indica que mientras en la entidad chiapaneca 45.78 por ciento de la población ocupada obtuvo hasta un salario mínimo, en los municipios que integran Los Altos 65.6 por ciento de ellos se ubicó en este nivel de ingreso, y si excluimos a San Cristóbal de Las Casas la cifra se eleva a casi 82 por ciento, lo que refleja una 
profunda asimetría donde lo rural y la condición étnica desempeñan un papel importante.

Dentro del ámbito de las comunidades estudiadas, la información muestra condiciones de alta precariedad en términos de los ingresos obtenidos por familia a través de tres fuentes: trabajo asalariado, subsidios del programa Oportunidades y recursos adquiridos por la vía del Procampo. En el siguiente cuadro se constata que estas tres fuentes sumaron al año un promedio de casi 17 mil pesos, lo que se complementa con los ingresos obtenidos por los escasos productos de la parcela.

\section{CUADRO 3}

Fuentes de ingreso por familia en el año (pesos)

\begin{tabular}{cc} 
Concepto & Ingreso anual por familia \\
\hline Ingresos por actividad laboral & 14982.50 \\
Ingresos por el programa Oportunidades & 6052.05 \\
Ingresos por el programa Procampo & 1127.77 \\
\hline Total & 22162.32
\end{tabular}

Fuente: Coporo, 2013.

En otras palabras, cada familia estaría recibiendo por la suma de las tres fuentes citadas 61 pesos diarios, equivalente a un salario mínimo. Ingreso que, no obstante, en muchos casos es mayor al que obtienen por la producción de sus minúsculas parcelas que comúnmente es para el autoconsumo.

\section{La migración en perspectiva en las localidades de Nichnamtic y Mukinaló}

A partir del contexto anterior es posible entender con mayor claridad la movilidad de la población en busca de medios de subsistencia. Chamula posee una larga historia de migración laboral interna, es un municipio que ha vivido desplazamientos debido a conflictos político-religiosos, pero también por la precariedad de sus recursos productivos y de empleo. Es también el ejemplo más nítido de una población que ha experimentado en su historia tres tipos de migración: interna, interestatal e internacional, por lo cual ha acumulado 
diversas experiencias de movilidad. Hoy, pese a que 99 por ciento de su población presenta condiciones de etnicidad, el municipio se conecta a los procesos globales a través de la venta de fuerza de trabajo barata, empleándose fundamentalmente en el sector de servicios, agricultura y construcción.

A continuación analizaremos el proceso migratorio a Estados Unidos en las comunidades de estudio, cuyo sustento son 123 cuestionarios aplicados en Nichnamtic y Mukinaló procesados con el programa SPSS, de manera complementaria se realizaron entrevistas a informantes calificados y la observación directa resultó de gran utilidad para dimensionar las condiciones de vida del migrante y su familia.

\section{Noticia histórica de la migración internacional en Chamula}

Una de las primeras cuestiones que interesaba establecer era la génesis de la migración internacional, de manera que a los encuestados se les preguntó el año en que salieron a Estados Unidos, los resultados mostraron que comenzaron a salir en 1993, fue Nichnamtic la primera localidad que tuvo migrantes internacionales. Para 1999 la migración internacional abarcó a las dos localidades y fue indicativo de que la crisis global se estaba reflejando en las comunidades indígenas de Chiapas, las cuales se encontraban al borde de la desesperación, hecho que se corroboraría en 1994 con el levantamiento del EZLN.

Mediante los encuestados se observaron distintos momentos en torno a las salidas, el periodo de mayor intensidad comprende de 1999 a 2007. A continuación se desglosan el año y número de salidas: en 1999 se registran 9, en 2000 el número asciende a 11, en los siguientes tres años a 9, en 2004 se contabilizaron 12, en 2005 se asienta la mayor cantidad de salidas con 25, en 2006 hay una drástica disminución a 6, en 2007 emigraron 10; a partir de este año y hasta el 2010 se observó una caída significativa que comenzó con 5 personas en 2008 y en los dos años posteriores sólo emigró 1 cada año. El comportamiento de los últimos tres años se corresponde con la crisis de la economía de Estados Unidos y el endurecimiento de los controles en la frontera. Como ya se ha indicado, en 1993 se registró el primer migrante, un niño de 12 años que fue invitado por un tío que vivía en la comunidad indígena La Candelaria, municipio de Huixtán, y quien permaneció 14 años en Estados Unidos.

Como parte de nuestra indagación importaba saber por qué ocurría la migración, y en este sentido las respuestas de los migrantes fueron muy claras: 59 
por ciento de los encuestados argumentó falta de trabajo y alrededor de 14 por ciento manifestó que ganaba muy poco, otro 6 por ciento declaró tener deudas. Así, casi 80 por ciento afirmó que por razones económicas. En el resto los motivos fueron diversos, por ejemplo, tener mejores condiciones de vida, desesperanza de vivir en la comunidad y la reunificación familiar. De esta manera, encontramos una historia migratoria muy interesante porque al cuestionar quiénes de la familia habían emigrado obtuvimos que 64 personas (52.4 por ciento) tenían familiares migrantes con distinto grado de parentesco: jefes de familia (28), esposas (23), hijos (5), hermanos (6), parientes (2). Cabe destacar la existencia de mujeres migrantes esposas, lo que se correlaciona con uno de los motivos de la migración que es justamente la reunificación familiar.

¿Cómo ocurre la migración a Estados Unidos? Esta es una pregunta fundamental que permite ver la existencia de la red migratoria en forma embrionaria. En las entrevistas aparecen tres actores clave: coyotes, ${ }^{11}$ familiares y amigos. En orden de jerarquía, de la muestra de 123 encuestados 58 (47.5 por ciento) manifestaron que fueron invitados por el coyote o pollero, 34 (27.6 por ciento) lo hicieron por una invitación familiar y 18 (14.6 por ciento) a través de amigos. En el resto, las respuestas fueron mixtas y otros no contestaron a la pregunta. A la luz de estos resultados debemos entender la importancia que para el momento tienen los polleros o coyotes; todo parece indicar que en principio son de otras comunidades de la región y luego en las mismas comunidades pasaron de migrantes a polleros (Coporo, 2013). De igual modo, se constata la función de la familia en el proceso migratorio y, finalmente, la intervención de los amigos refleja la trascendencia del capital social (Bourdieu, 1980; Coleman, 1990; Piselli, 2003) y la formación de una red migratoria (Granovetter, 1973) que facilita la migración presente en el momento en que ocurre la mayor salida de migrantes, una vez formada la red básica de contactos.

Un aspecto central en los hallazgos de la investigación de campo fue que la crisis de 2008 afectó a la economía de los polleros, algunos quedaron endeudados con grandes cantidades de dinero, incluso perdieron sus propiedades, otros han sido apresados acusados de tráfico de personas y otros están prófugos; suponemos que esto es así a causa de la disminución de la migración y porque algunos ofrecían crédito a los migrantes. En una entrevista, una esposa de pollero comentó que su esposo primero fue migrante y después pollero, pero que por esa

${ }^{11}$ En estricto sentido se refieren al pollero. 
actividad fue apresado, después lo dejaron en libertad pero permanece escondido porque todavía debe dinero (Coporo, 2013).

\section{De Chamula a Estados Unidos}

¿Cómo definen el lugar de destino los migrantes de Los Altos? Sin lugar a dudas los coyotes, los familiares y los amigos que conforman la red migratoria son determinantes en la definición del lugar de destino de los migrantes. La respuesta de los 123 encuestados revela la preponderancia de tres estados como lugar de llegada: en primer lugar Florida, en segundo Georgia y en tercero Alabama. Se trata de un espacio bastante compacto donde parece moverse la población indígena de Chamula. A Florida se fueron 59, a Georgia 15 y a Alabama 12, el resto se distribuyó en los estados de California, Arizona y Washington. De forma más específica, los que se fueron a Florida se concentraron en Tampa, por eso no es una casualidad que allí se haya formado la «pequeña Chamula», Miami aparece como segundo lugar de destino; en Georgia sobresale Cairo y Atlanta; finalmente los que emigraron a Alabama no mencionaron un lugar específico. Adicionalmente, es importante señalar que 33.3 por ciento de los encuestados fueron esperados por un familiar, 14.6 por amigos y 16.3 por ciento manifestaron que no tuvieron quien los recibiera.

La información de campo se correlaciona con las estadísticas de Matrículas Consulares de Alta Seguridad. Para 2012 los migrantes chamulas realizaron el trámite en los siguientes estados: en primer lugar aparece Florida con 298 matrículas expedidas en Orlando y 44 en Miami; en segundo lugar figura Atlanta con 33 matrículas tramitadas. Aunque para el conjunto del estado de Chiapas la expedición de matrículas se amplió: 45 por ciento fueron otorgadas, en orden de importancia, por los consulados de Atlanta, Raleigh, Orlando, Los Ángeles y Miami. ${ }^{12}$

\section{Empleo y salario en el lugar de destino}

La información de campo refleja una correlación positiva entre las actividades que realizaban los migrantes en la comunidad y las llevadas a cabo en el lugar

${ }^{12}$ Véase Instituto de los Mexicanos en el Exterior, en: http://www.ime.gob.mx/es/agenda-de-informacion/ estadisticas-de-mexicanos-en-el-exterior 
de destino. De los 123 encuestados, 63 se emplearon en actividades de jardinería (13 indicaron que trabajaron en viveros, "nursería» como coloquialmente lo expresan), 17 en la construcción y 8 en empacadoras de pollo. El resto declaró haber trabajado en agricultura, carpintería, lavandería, restaurante y refaccionaria. Su actividad principal en la comunidad de origen estaba orientada a la agricultura y ello explica que su empleo en Estados Unidos se relacione de forma preponderante con mantenimiento de jardines. Se trata, finalmente, de actividades precarias que se corresponden con bajos salarios.

En efecto, de la muestra de encuestados, 73 (60 por ciento) manifestaron haber ganado entre 6 y 8.5 dólares por hora; 28 (22.7 por ciento) declararon ingresos entre 9 y 11.5 dólares; 5 un ingreso de entre 12 y 26.5 dólares; y solamente 8 indicaron haber ganado entre 3 y 5.5 dólares por hora, suponemos que se trata de los casos que se emplearon por primera vez y que no tenían contactos previos. En medio de la precariedad laboral, la diferencia salarial entre México y Estados Unidos es abismal, por lo que el espejismo del dólar constituye un factor en la decisión de emigrar. Para ilustrar esa diferencia tomemos como referencia el año 2005, que es cuando se registró la mayor migración en las comunidades de estudio: el promedio de las tres áreas geográficas en que se divide el país para la fijación del salario mínimo es de 45.4 pesos diarios, que multiplicado por treinta días daría un salario mensual de mil 362 pesos. Por otro lado, en el mismo año el dólar costaba 10.62,13 que multiplicado por 7 dólares, que es el pago promedio por hora de la mayoría de los encuestados, resultaría 74.34 pesos por hora, 594.72 pesos por día y 17,841.6 pesos mensuales. Es decir, un trabajador mexicano ganaría en Estados Unidos en dos días lo que en el país le llevaría un mes.

\section{Ahorro y remesas familiares}

Mucho se ha discutido sobre la importancia de las remesas familiares, el debate se ha centrado sobre sus efectos en el desarrollo; no se habla, sin embargo, de lo que implica para el migrante el envío del dinero a su lugar de origen, el ahorro forzoso. A final de cuentas, la información de campo en las comunidades de estudio evidencian algo que ha sido desmitificado por algunos estudiosos (Delgado y Márquez, 2007; Delgado, Márquez y Rodríguez, 2009), pues las remesas no generan desarrollo, en las comunidades no se observan cambios sustanciales 
en la calidad de vida de sus habitantes. Aunque debemos admitir que en algunas localidades de Chamula la «arquitectura» de la migración es impactante, casas de dos y tres pisos, algunas de estilo californiano; por la información obtenida en campo se sabe que en la mayoría de los casos se trata de propiedades de polleros.

Complementariamente, preguntamos a los encuestados sobre las cantidades que logran ahorrar y sus envíos de dinero a sus familias. Obtuvimos 114 respuestas con amplia variación en las cantidades, pero los más numerosos es posible agruparlos de la siguiente manera: 13 migrantes pudieron ahorrar 500 dólares al mes, 15 ahorraron 400 dólares, 12 ahorraron 300 dólares, 8 ahorraron 200 dólares y 10 guardaron 100 dólares. En síntesis, este grupo de 58 migrantes generaron un ahorro que va de 100 a 500 dólares. De modo paralelo, encontramos un grupo de 31 migrantes que afirmó haber ahorrado entre 531 y 2 mil dólares mensuales. Cabe destacar que dentro de este conjunto observamos un segmento de 19 migrantes que ahorró entre mil y 2 mil dólares, hecho que contrasta notablemente con otro grupo pequeño de seis migrantes, que se ubicó en el extremo con un ahorro entre 4 y 44 dólares mensuales. Para explicarlo en términos más técnicos: del total de respuestas, la media fue de 487.5 dólares; la mediana y la moda de 400 dólares; y la desviación típica de 411.3 dólares, lo que significa que el conjunto de valores se concentraron en torno a la media. Prevalece la duda sobre el grupo que declaró haber ahorrado entre mil y 2 mil dólares mensuales, tal acontecmiento parece poco créble a no ser que trabajaran doble turno, con un salario por arriba del mínimo, pues lo máximo que podrían ganar en un mes — con un salario mínimo de 7 dólares la hora — sería de 18 mil pesos, ${ }^{14}$ a lo que habría que descontar la manutención.

Entre el ahorro y el envío de dinero se constatan diferencias que creemos se vinculan con las estrategias individuales y familiares para el pago de deudas y la sobrevivencia. ${ }^{15}$ Un primer grupo de nueve encuestados declaró haber enviado entre 40 y 76 dólares, ${ }^{16}$ un segundo grupo de 28 confirmó haber enviado entre 80 y 135 dólares, un tercero entre 161 y 309 dólares y el último de 37 encuestados

\footnotetext{
${ }^{14}$ Suponiendo que trabajaran una jornada de ocho horas y teniendo en cuenta el tipo de cambio que para el año 2005 estaba en 10.62 pesos por dólar.

${ }^{15}$ La mayoría de los encuestados proporcionaron datos de envíos en pesos, sólo algunos en dólares, por lo que se optó por presentar la información en pesos.

${ }^{16}$ Los valores obtenidos en la encuesta directa se convirtieron en dólares, pese a que la mayoría de los migrantes mencionó el dato en pesos. El tipo de cambio utilizado de 2010 del Banco de México.
} 
entre 323 y mil 212 dólares, el resto de los envíos son poco significativos pues son cantidades inferiores a 40 dólares.

Los datos presentan una amplia dispersión que van de 0 a mil 212 dólares; sin embargo, el valor promedio de envío de remesas es de 202 dólares mensuales. No obstante lo anterior, la moda o el envío de remesas más frecuente fue de 81 dólares. Por otro lado, la desviación estándar de 287 dólares indica que los envíos más consistentes se encuentran en el rango de 0 hasta 489 dólares: respecto a la frecuencia, 50 por ciento lo hacía cada mes, y la otra mitad entre uno y seis meses; la mayor frecuencia se hizo mensualmente (45 por ciento de los encuestados). La desviación estándar de 114 se concentró en el rango de una semana a tres meses.

Si consideramos que el promedio de envío de remesas es 202 dólares por mes, esto no puede traducirse en lo que se ha dado en llamar «desarrollo». Además, deben tomarse en cuenta los pagos al pollero o coyote o al prestamista que financió el viaje, cuyo cobro de intereses oscila entre 10 y 20 por ciento mensual. Si el migrante tuvo que pedir 30 mil pesos y si la tasa de interés promedio es de 15 por ciento mensual, en teoría tendría que pagar 4 mil 500 pesos mensuales (363 dólares) por lo menos durante medio año, a ello debe añadirse el pago del monto principal, que agregaría medio año más. Lo más seguro es que el migrante no pueda pagar en un año, lo que implica que por lo menos tendría que estar dos años en Estados Unidos para terminar de pagar sus deudas. El espejismo del dólar hace ver cosas distintas - a pesar de lo real que resulta la diferencia salarial entre México y Estados Unidos-, pues no permite ver el costo económico y social, el riesgo y la imposibilidad de aspirar a la seguridad social en aquel país. Dichos elementos cuestionan los beneficios de los dólares de la migración traducidos en desarrollo.

En ese sentido, es prioritario advertir que 81.3 por ciento de los encuestados usa las remesas para pagar la deuda del préstamo, es evidente entonces que la migración en tal etapa sólo sirve para salir de las deudas contraídas. El resto (18.7 por ciento) mostró que además de pagar el préstamo sirven para financiar diferentes actividades: alimentación, cooperaciones religiosas, arreglo de la casa, pago de deudas atrasadas, pago de cargos, compra de terrenos, animales y artículos electrodomésticos.

La información de los encuestados es congruente con las necesidades de permanecer un tiempo razonable para pagar las deudas y lograr ciertos objetivos que motivaron el viaje. De los 123 encuestados, 47.2 por ciento permaneció en 
Estados Unidos de 2 a 3.5 años; 22 por ciento de 4 a 5.5 años; 21.1 por ciento hasta año y medio; 5.7 por ciento de 6 a 7.5 años. Asimismo, se perciben dos grupos muy reducidos que se afincaron de 8 a 9.5 años, y otro de 12 a 13.5 años ( 0.8 por ciento, respectivamente). Sólo tres no respondieron a la pregunta sobre el tiempo de permanencia en la Unión Americana. En adición, 82.9 por ciento de los encuestados declaró haber regresado a su comunidad en una sola ocasión durante el periodo de estancia en Estados Unidos, situación que demostraría la ausencia de una migración de tipo circular.

\section{El deseo de regresar a Estados Unidos}

Si bien los logros alcanzados con la migración son escasos, un porcentaje nada despreciable de los migrantes retornados declaró su interés por regresar a Estados Unidos; de los 123 encuestados, 46 (37.4 por ciento) brindaron razones que reflejan su condición de precariedad. Otro grupo muy pequeño externó sus aspiraciones más allá de la subsistencia: 15 expresaron que aquí no había trabajo, 8 que tenían deudas, 6 que no tenían dinero, 8 manifestaron su deseo por tener dinero, el resto esgrimió distintos argumentos (mantener a la familia, tener un carro propio, construir una vivienda, solventar los estudios de los hijos). En las respuestas predominan los motivos laborales y la necesidad de obtener dinero; sin embargo, desconcierta el hecho de que otros siguen pensando que su estancia en Estados Unidos les permitirá cumplir con sus metas.

\section{Conclusiones}

En el año 2000, Stephen Castles afirmó:

No existe una relación de causa a efecto entre la pobreza y la emigración. La emigración desde zonas muy pobres suele ser muy poco común, porque las personas carecen de capital económico necesario para viajar, del capital cultural necesario para tomar conciencia de las oportunidades que hay en otros lugares y del capital social que se necesita para lograr encontrar trabajo y hacer frente al nuevo medio (21). 
Años más tarde, en 2004, Castles y Miller sostenían que uno de los temas de gran trascendencia para los países de destino es la regulación de las migraciones (24). ìPor qué cobra centralidad la regulación? Creemos que existen varias razones, entre ellas el crecimiento de la migración, pues ni el «sellamiento» de las fronteras ni las deportaciones ha logrado detenerlo. Incluso es posible enumerar otras preocupaciones: la existencia de una población redundante, el incremento de la pobreza, el fin del Ejército Industrial de Reserva, la presión que ejercen los migrantes en el país de destino, la supuesta amenaza a la unidad e identidad nacional, el racismo, la seguridad nacional, y más recientemente la crisis económica y laboral que desde 2008 no ha podido ser superada.

A la luz de los resultados de trabajo de campo y de otros estudios realizados en Chiapas que revelan el mismo escenario planteado con anterioridad (Villafuerte, 2013), en el marco de los cambios que se han venido dando en el capitalismo global a partir del siglo XXI, es preciso revisar el vínculo pobreza y migración, sobre todo porque la pobreza se ha globalizado y la crisis de empleo se ha agudizado. De ser correcta la tesis de Castles, a estas alturas del milenio los menos pobres ya emigraron y ahora toca el turno a los más pobres que han logrado acumular capital social y constituir redes migratorias, como es el caso estudiado.

La migración internacional en el municipio de Chamula es un síntoma inequívoco de las transformaciones provocadas por el mercado. La comunidad indígena-campesina en las últimas dos décadas ha sufrido drásticos cambios: sus economías no generan los medios de subsistencia, no sólo por el crecimiento natural de su población, que sigue siendo alto en comparación con la sociedad urbana, sino también por el agotamiento de sus precarios recursos productivos, así como el cambio en el patrón de consumo.

Adicionalmente, en los últimos 25 años hemos observado que Chamula, que en algún momento se caracterizó por cerrado y refractario a las transformaciones que ocurrían en otros espacios dentro y fuera de Chiapas, exhibe diversos cambios. En principio, la fragmentación del modelo de la Comunidad Revolucionaria Institucional, que bien distinguió Rus (1998). En seguida, la aceptación, no sin dificultades, de grupos religiosos diferentes al catolicismo, en la actualidad en ciertas comunidades del municipio se aprecian templos evangélicos. Posteriormente, el Partido Revolucionario Institucional, como partido único, empieza a perder hegemonía, ahora se observa mayor presencia de los partidos Acción Nacional y de la Revolución Democrática, pero además los representantes de cada 
partido son descendientes de familias no arraigadas al municipio, aunque mantienen sus derechos. Finalmente es indudable que diversos programas de combate a la pobreza como el de Oportunidades, están transformando normas y valores comunitarios, propios de la persistencia cultural.

En la década de los 1990 se visibilizan varios problemas sociales en el municipio de Chamula, por ejemplo, emerge la migración internacional, se diversifica la oferta política y varían las opciones religiosas pese a las expulsiones. De modo simultáneo comienzan a manifestarse los efectos de las políticas neoliberales en el campo chiapaneco, implementadas en México desde la década de los 1980; incluso en ese momento surgen los estragos de la crisis del café y en general la disminución de los precios internacionales de los principales productos agrícolas que impactan en el mercado laboral tradicional.

Existe también en los 1990 una nueva generación de jóvenes que apareció desde mediados de los 1980, pero cuyo contexto además de la comunidad rural, es la ciudad, fundamentalmente San Cristóbal de Las Casas. De esa generación destacan sobre todo los hijos de líderes y dirigentes comunitarios, aquellos líderes (profesores) que institucionalizaron al PRI en la comunidad, sobresalen de igual forma los hijos de los protestantes y de los migrantes internos. En el primer caso encontramos a Víctor y su familia (permaneció en Estados Unidos durante 14 años), quien se fue a los 12 años y regresó hace tres. Su papá es profesor y fue presidente municipal de Chamula. Este es un ejemplo de que los jóvenes retornados incentivan el cambio social en la comunidad de origen, mediante su práctica cotidiana con los nuevos parámetros culturales incorporados. En el campo se observa una marcada estratificación social familiar, entre quienes tuvieron «éxito en la migración» y los que no han salido, puesto que sirve de ejemplo para quienes piensan emigrar.

\section{Referencias}

Angulo, Jorge Ignacio (2007), "Crisis y nuevas tendencias migratorias en los campesinos de Chiapas; el caso de la Sierra", Asociación para el Fomento de los Estudios Históricos en Centroamérica (30), en http://www.afehc-historia-centroamericana.org/ index.php?action $=$ fi_aff\&id $=1484$

Betancourt, Darío (1977), Bases regionales de las comunas urbanas en San Cristóbal de Las Casas, Chiapas, Chiapas, Universidad Autónoma de Chiapas. 
Bourdieu, Pierre (1980), "Le capital social, notes provisoires», Actes de la recherche en sciences sociales, 31(1), pp. 2-3, en http://www.persee.fr/web/revues/home/prescript/article/arss_0335-5322_1980_num_31_1_2069

Castles, Stephen (2000), "Migración internacional a comienzos del siglo XXI: tendencias y problemas mundiales», Revista Internacional de Ciencias Sociales (165), pp. 1732, en http://unesdoc.unesco.org/images/0012/001238/123852s.pdf

Castles, Stephen y Mark Miller (2004), La era de la migración. Movimientos internacionales de población en el mundo moderno, México, Instituto Nacional de Migración/Cámara de Diputados LIX Legislatura/Universidad Autónoma de Zacatecas/Fundación Colosio/Miguel Ángel Porrúa.

Consejo Nacional de Evaluación de la Política de Desarrollo Social (Coneval) (2010), "Información por municipio. Chamula. Chiapas», en http://www.coneval.gob.mx/ cmsconeval/rw/pages/medicion/multidimencional/informacion_municipios. es.do

(2012), "Informe de pobreza y evaluación en el estado de Chiapas 2012», en http://www.coneval.gob.mx/coordinacion/entidades/Documents/Chiapas/ principal/07informe2012.pdf

Coporo, Gonzalo (2013), «Migración, pobreza y desarrollo: estudio de casos en dos localidades del municipio de Chamula en Los Altos de Chiapas» (tesis doctoral), Centro de Estudios Superiores de México y Centroamérica, Universidad de Ciencias y Arte de Chiapas.

Delgado, Raúl y Humberto Márquez (2007), «Repensar la relación entre migración y desarrollo a partir del caso de México», LiminaR, 5(2), pp. 8-25.

Delgado, Raúl, Humberto Márquez y Héctor Rodríguez (2009), «Seis tesis para desmitificar el nexo entre migración y desarrollo», Migración y Desarrollo, 7(12), pp. 27-52.

García de León, Antonio (1985), Resistencia y utopía. Memorial de agravios y crónicas de revueltas y profecías acaecidas en la provincia de Chiapas durante los últimos 500 años de su historia, México, Era.

Granovetter, Mark S. (1973), «La fuerza de los vínculos débiles», American Journal of Sociology, 78(6), pp. 1360-1380.

Instituto Nacional de Estadística Geografía e Informática (INEGI) (2010), «Principales resultados del censo de población y vivienda 2010. Chiapas», en http://www.inegi. org.mx/prod_serv/contenidos/espanol/bvinegi/productos/censos/poblacion/ 2010/princi_result/chis/07_principales_resultados_cpv2010.pdf 
(2000), "Perfil sociodemográfico. Chiapas», en http://www.inegi.org.mx/ prod_serv/contenidos/espanol/bvinegi/productos/censos/poblacion/2000/ perfiles/perfil_chiapas-1.pdf

(2007), «Panorama agropecuario en Chiapas», en http://www3.inegi.org.mx/ sistemas/biblioteca/ficha.aspx? upc $=702825003981$

López, Antonio (1992), «Sistema religioso-político y las expulsiones en Chamula, Chiapas, México» (tesis de licenciatura), Escuela de Ciencias Sociales, Universidad Autónoma de Chiapas.

López, Omar, Julio César Molina y Daniel Villafuerte (2010), "Apuntes sobre las nuevas migraciones en San Cristóbal de Las Casas, Chiapas», en Anuario de Estudios Indígenas XIV. Migraciones, ciudades y cambio cultural, pp. 117-152, San Cristóbal de Las Casas, Instituto de Estudios Indígenas-Universidad Autónoma de Chiapas.

Marroquín, Alejandro (1978), La ciudad mercado (Tlaxiaco), México, Instituto Nacional Indigenista.

Morquecho, Gaspar (1992), Los indios en un proceso de organización. La organización indigena de Los Altos de Chiapas, ORIACH (tesis de licenciatura), Escuela de Ciencias Sociales, Universidad Autónoma de Chiapas.

Paniagua, Alicia (1983), "Chiapas en la coyuntura centroamericana», Cuadernos Politicos (38), pp. 36-54, en http://www.bolivare.unam.mx/cuadernos/cuadernos/contenido/CP.38/CP38.5AliciaPaniagua.pdf

Pérez, María Isabel (1994), Expulsiones indígenas. Religión y migración en tres municipios de los Altos de Chiapas, Chenalhó, Larrainzar y Chamula, Ciudad de México, Claves Latinoamericanas.

Perezgrovas, Raúl (1999), Los carneros de San Juan, San Cristóbal de Las Casas, Chiapas, Chiapas, Instituto de Estudios Indígenas-Universidad Autónoma de Chiapas.

Piselli, Fortunata (2003), "Capital social: un concepto situacional y dinámico», en Bagnasco, Arnaldo, Fortunata Piselli, Alejandro Pizzorno y Carlo Trigilia, El capital social. Instrucciones de uso, pp. 53-87, México, Fondo de Cultura Económica.

Pozas, Ricardo (1948), Juan Pérez Jolote: biografía de un Tzotzil, México, Fondo de Cultura Económica.

Rus, Diana y Jan Rus (2008), "La migración de trabajadores indígenas de Los Altos de Chiapas a Estados Unidos, 2001-2005: el caso de San Juan Chamula», en Villafuerte, Daniel y María del Carmen García (coords.), Migraciones en el sur de México y Centroamérica, pp. 333-382, Universidad de Ciencias y Artes de Chiapas/Miguel Ángel Porrúa. 
Rus, Jan (1998), «La comunidad revolucionaria institucional: la subversión del gobierno indígena en los Altos de Chiapas, 1936-1968», en Viqueira, Juan Pedro y Mario Humberto Rus (eds.), Chiapas. Los rumbos de otra historia, pp. 251-277, México, Centro de Investigaciones y Estudios Superiores en Antropología Social-Universidad Nacional Autónoma de México/Centro de Estudios Mexicanos y Centroamericanos-Universidad Autónoma de Guadalajara.

Rus, Jan (2009), "La lucha contra los caciques indígenas en Los Altos de Chiapas: disidencia, religión y exilio en Chamula, 1965-1977», en Anuario de Estudios Indígenas XIII. Antropología del poder, pp. 181-230, San Cristóbal de Las Casas, Instituto de Estudios Indígenas-Universidad Autónoma de Chiapas.

Velasco, Manuel (1972), Chiapas. Segundo Informe de Gobierno, Tuxtla Gutiérrez, Chiapas, México, Libros de México.

Villafuerte, Daniel (2013), «La catástrofe neoliberal en Chiapas: pobreza, precarización laboral y migraciones», en Baltar, Enrique, María da Gloria Marroni y Daniel Villafuerte (coords.), Viejas y nuevas migraciones forzadas en el sur de México, Centroamérica y el Caribe, pp. 215-248, México, Sitesa Editores. 